\title{
COMPARAÇÃO ENTRE OS AJUSTES DE CURVA EXPONENCIAL E POLINOMIAL NA DETERMINAÇÃO DO LIMIAR DE LACTATO PELO MÉTODO DMAX
}

\author{
Dra. FABIANA ANDRADE MACHADO \\ Professora do Departamento de Educação Física, Universidade Estadual de Maringá \\ (Maringá - Paraná - Brasil) \\ E-mail: famachado_uem@hotmail.com \\ MS. WONDER PASSONI HIGINO \\ Professor da Faculdade de Educação Física, Centro Universitário Católico Salesiano Auxilium \\ (Lins - São Paulo - Brasil) \\ E-mail:wonderhigino@ig.com.br \\ GRAD. CECÍLIA SEGABINAZI PESERICO \\ Mestranda em Educação Física, Programa Associado UEM/UEL \\ (Maringá - Paraná - Brasil) \\ E-mail: ceciliapeserico@gmail.com \\ GRAD. PAULO VICTOR MEZZAROBA \\ Mestrando em Educação Física, Programa Associado UEM/UEL \\ (Maringá - Paraná - Brasil) \\ E-mail: paulomezzaroba@hotmail.com

\section{Dra. SOLANGE MARTA FRANZÓI DE MORAES} \\ Professora do Departamento de Ciências Fisiológicas, Universidade Estadual de Maringá \\ (Maringá - Paraná - Brasil) \\ E-mail:smfmoraes@gmail.com
}

\begin{abstract}
RESUMO
Comparou-se a velocidade de corrida no limiar de lactato (vLL) determinada pelo método Dmax utilizando-se os ajustes de curva exponencial mais constante ( $\left.V L L_{\text {exp }}\right)$ e polinomial de terceira ordem $\left(v L L_{p o}\right)$. Dezessete corredoras recreacionais realizaram um teste incremental descontínuo em esteira rolante até a exaustão voluntária com coleta de sangue entre os estágios para análise do lactato. $A v L L_{E \times p}(10,9 \pm 0,8 \mathrm{~km} / \mathrm{h})$ foi estatisticamente diferente ( $P$ $<0,05)$ da vLL $L_{P o l}(10,4 \pm 1,1 \mathrm{~km} / \mathrm{h})$ utilizando-se o teste $t$ de Student pareado. A correlação entre $a v L L_{E x p}$ e $a v L L_{P o l}$ foi de $r=0,84$. As correlações entre a velocidade pico e as $v L L$ foram $r=0,94\left(v L L_{\text {exp }}\right)$ e $r=0,69\left(v L L_{\text {po }}\right)$. Foi concluído que a escolha da curva de regressão do lactato sanguíneo influi no valor da $v L L$, sendo a $v L_{\text {pol }}$ consistentemente inferior à $v L L_{\text {exp }}$.
\end{abstract}

PALAVRAS-CHAVE: Equação; método do máximo desvio; velocidade de corrida; regressão. 
O limiar de lactato (LL) é considerado um indicador da aptidão aeróbia que tem sido muito utilizado por treinadores, atletas e pesquisadores para a predição do desempenho, prescrição da intensidade de exercício e avaliação dos efeitos do treinamento (BILLAT, 1996; PAPADOPOULOS; DOYLE; LABUDDE, 2006; STRATTON et al., 2009).

Para a sua determinação, diversos métodos foram propostos, encontrandose algumas vezes diferentes estimativas da intensidade referente à sua ocorrência (DAVIS et al., 2007; FAUDE; KINDERMANN; MEYER, 2009; THOMAS et al., 2008; TOKMAKIDIS; LÉGER; PILIANIDIS, 1998). Entre esses métodos, o método do máximo desvio (Dmax) proposto por Cheng et al. (1992), que depende tanto do ajuste dos dados à curva lactato-intensidade derivadas de um teste incremental máximo como dos pontos inicial e final desta curva, além de objetivo, contrapondo-se à detecção visual do LL, possui a vantagem de considerar as características individuais na determinação deste parâmetro, diferentemente de outros métodos, como o método da concentração fixa do lactato, que pode subestimar ou superestimar a sua determinação. (TOKMAKIDIS; LÉGER; PILIANIDIS, 1998). No entanto, não existe ainda um consenso entre autores sobre qual função deva ser utilizada para o ajuste dos dados à curva lactato-intensidade na aplicação deste método.

Cheng et al. ( 1992) utilizaram a curva polinomial de terceira ordem para o ajuste dos dados. No entanto, não existe nenhum estudo na literatura que tenha demonstrado que a curva que representa o aumento na concentração de lactato em função do aumento da intensidade de exercício apresenta uma resposta polinomial de terceira ordem. Por outro lado, Hughson, Weisiger e Swanson ( 1 987) demonstraram que a curva exponencial mais constante pode representar a resposta fisiológica do lactato durante um teste progressivo, visto que esta se ajustou muito bem à curva lactato-intensidade. Nicholson e Sleivert (200l) utilizaram a função exponencial mais constante para o ajuste dos dados no estudo em que a velocidade de corrida no limiar de lactato ( $v L L$ ) determinada pelo método Dmax apresentou uma forte correlação com a velocidade de corrida de $10 \mathrm{~km}(r=0,86$; $P<0,00 \mathrm{I})$. Já Bishop, Jenkins e Mackinnon ( 1998 ) utilizaram a função polinomial de terceira ordem em um estudo que verificou que o Dmax, entre seis índices de lactato comumente utilizados, foi o que obteve a maior correlação com o desempenho em provas de I hora no ciclismo ( $r=0,84 ; P<0,00$ I). Por sua vez, Czuba et al. (2009) não fizeram referência à função utilizada no estudo em que encontraram uma forte correlação em ciclistas bem treinados entre a carga no LL obtida pelo método Dmax e a carga da máxima fase estável do lactato sanguíneo $(r=0,97 ; P<0,00$ I $)$. 
Deste modo, considerando a inexistência de um consenso sobre qual função deva ser utilizada para a determinação da vLL pelo método Dmax, e sabendo-se que a escolha da função para o ajuste dos dados provavelmente influenciará na estimativa do LL, o objetivo deste estudo foi comparar os ajustes exponencial mais constante e polinomial de terceira ordem na determinação da $v L L$ pelo método Dmax. Além disso, a $v L L$ determinada por ambos os ajustes foi comparada com a velocidade pico (Vpico) atingida ao final do teste incremental máximo, visto ser esta uma variável preditora do desempenho de média e longa distâncias (NOAKES; MYBURGH; SCHALL, 1990; SCOTT; HOUMARD, 1994; STRATTON et al., 2009).

\section{MATERIAL E MÉTODOS}

\section{SUJEITOS}

Participaram deste estudo 17 corredoras recreacionais, aparentemente saudáveis, integrantes de grupos de corrida da cidade. As corredoras foram selecionadas seguindo os seguintes critérios: experiência mínima na modalidade (dois anos) e regularidade de treinamento (pelo menos duas vezes por semana). As características antropométricas das participantes foram: idade 42,I \pm 6,7 anos; estatura I,63 \pm 0,03 m; massa corporal 57,2 \pm 4,9 kg; e índice de massa corporal (IMC) 21,5 \pm $1,8 \mathrm{~kg} / \mathrm{m}^{2}$. As participantes possuíam experiência de 3,1 $\pm 1,9$ anos de treinamento com freqüência de 2,6 \pm 0,5 sessões de treinamento semanais percorrendo uma distância semanal de 25,9 $\pm 6,0 \mathrm{~km}$. Todas as participantes leram e assinaram o termo de consentimento livre e esclarecido antes do início dos testes. O protocolo utilizado neste estudo foi previamente aprovado pelo Comitê de Ética local (\# 719/2010).

\section{TESTE INCREMENTAL EM ESTEIRA ROLANTE}

O teste incremental foi realizado em uma esteira rolante (INBRASPORT Super ATL, Porto Alegre, Brasil). A velocidade inicial foi de $7 \mathrm{~km} / \mathrm{h}$ com incrementos de I km/h a cada 3 minutos, após um prévio aquecimento de 5 minutos a $5 \mathrm{~km} / \mathrm{h}$. A inclinação da esteira foi mantida em $1 \%$ durante todo o teste. Entre os estágios houve pausa de 30 segundos para a coleta de $25 \mu \mathrm{L}$ de sangue do lóbulo da orelha para análise da concentração de lactato sanguíneo através de um analisador eletroquímico (YSI I 500, Ohio, EUA). As participantes foram orientadas a se absterem da prática de exercícios físicos vigorosos e da ingestão de cafeína ou bebidas alcoólicas 48 horas antes do teste. Além disso, foram orientadas a comparecerem ao laboratório bem hidratadas e a realizarem a última refeição com antecedência mínima de 2 horas da realização do teste. As variáveis cardiorrespiratórias foram continuamente medidas 
utilizando um analisador metabólico de gases (MedGraphics VO2000, St. Paul, USA), calibrado antes de cada teste de acordo com as instruções do fabricante. A freqüência cardíaca (FC) foi constantemente monitorada (Polar, Kempele, Finlândia). A percepção subjetiva de esforço (PSE) foi verificada ao final de cada estágio ao longo do teste incremental através da escala de Borg 6-20 (BORG, 1982). O teste foi mantido até a exaustão voluntária, sendo que cada participante foi encorajada verbalmente a se manter em exercício pelo maior tempo possível. O esforço máximo foi considerado atingido se pelo menos três dos seguintes critérios fossem observados: I) platô no consumo de oxigênio $\left(\mathrm{VO}_{2}\right)$ com o aumento da velocidade $\left(\Delta \mathrm{VO}_{2} \leq 150 \mathrm{~mL} / \mathrm{min}\right)$; 2) razão de trocas respiratórias $(R E R) \geq I, 15 ; 3)$ concentração de lactato sanguíneo ao final do teste ([La]pico) $\geq 8 \mathrm{mM}$; 4) frequência cardíaca pico (FCpico) entre \pm 10 bpm da FC máxima predita pela idade (220-idade) e 5) PSE > 18 na escala de Borg 6-20 (HOWLEY; BASSETT; WELCH, 1995).

\section{AJUSTE DOS DADOS: CURVA EXPONENCIAL MAIS CONSTANTE}

A curva exponencial mais constante é representada pela seguinte equação: $[\mathrm{La}]=a+b \cdot e^{(c \cdot v)}$

em que, e é o número neperiano; $v$ é a velocidade em $\mathrm{km} / \mathrm{h}$ durante o teste incremental na esteira rolante; $a, b$ e c são os parâmetros da curva; e [La] é o valor predito da concentração de lactato em função da velocidade. Os parâmetros $a, b$ e c foram determinados com o auxílio do Statistical Package for the Social Sciences (SPSS Inc. USA), versão 17.0.

\section{AJUSTE DOS DADOS: CURVA POLINOMIAL DE TERCEIRA ORDEM}

A curva polinomial de terceira ordem é representada pela seguinte equação: $[\mathrm{La}]=a+b \cdot v+c \cdot v^{2}+d \cdot v^{3}$

em que, $v$ é a velocidade em $\mathrm{km} / \mathrm{h}$ durante $\mathrm{o}$ teste incremental na esteira rolante; $\boldsymbol{a}, \boldsymbol{b}, \boldsymbol{c}$ e d são os parâmetros da curva; e [La] é o valor predito da concentração de lactato em função da velocidade. Os parâmetros $a, b, c$ e d foram determinados com o auxílio do Statistical Package for the Social Sciences (SPSS Inc. USA), versão 17.0.

\section{DETERMINAÇÃO DA VLL PELO MÉTODO DMAX}

O LL foi determinado pelo método Dmax proposto por Cheng et al. ( 1992). O método Dmax consiste na determinação do ponto em uma curva ajustada do 
lactato sanguíneo pela intensidade de exercício, no qual ocorre a maior distância perpendicular da linha traçada conectando o primeiro e o último ponto desta curva. A Figura I apresenta este ponto, tanto para um ajuste exponencial mais constante (A) como para um ajuste polinomial de terceira ordem (B).
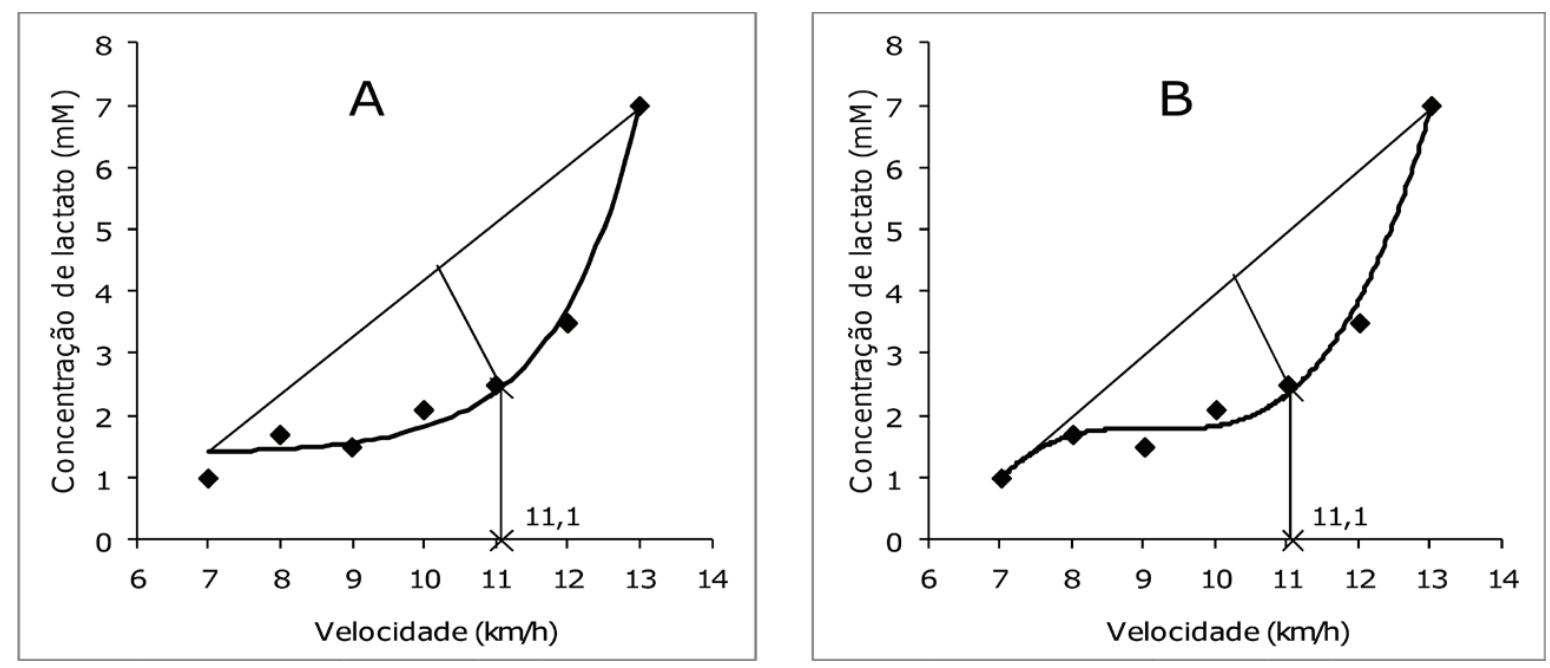

Figura I: Método Dmax para o ajuste exponencial mais constante $(A)$ e polinomial de terceira ordem (B). Ambos os métodos estão apresentados para um mesmo conjunto de dados fictícios e resultaram na velocidade no limiar de lactato de $11, \mathrm{l} \mathrm{km} / \mathrm{h}$.

A máxima distância perpendicular que representa a $v L L$ determinada pelo método Dmax ocorre no ponto em que a tangente da curva ajustada é igual ao coeficiente angular da reta traçada conectando as duas extremidades desta curva (ZHOU; WESTON, 1997). Considerando-se que a tangente de uma curva é obtida através da primeira derivada de sua função, após alguns procedimentos matemáticos, resulta-se nas seguintes expressões do LL:

$$
v L L_{\text {Exp }}=\left\{\ln \left[\left(e^{((\cdot \cdot \cdot)}-e^{(c \cdot v i)}\right) /(c \cdot v f-c \cdot v i)\right]\right\} / c
$$

em que, e é o número neperiano; c é o parâmetro da curva exponencial mais constante; vi e vf são respectivamente as velocidades inicial e final do teste incremental.

$$
v L L_{\text {Pol }}=\left\{-c+\left[\left(c^{2}-3 \cdot d \cdot(b-\Delta)\right]^{1 / 2}\right\} /(3 \cdot d)\right.
$$

em que, $b, c$ e $d$ são os parâmetros da curva polinomial de terceira ordem. Delta $(\Delta)$ é o coeficiente angular da reta traçada conectando as duas extremidades desta curva, ou seja:

$$
\Delta=\left([\mathrm{La}]_{\text {final }}-[\mathrm{La}]_{\text {inicial }}\right) /(\mathrm{vf}-\mathrm{vi})
$$

em que, $[\mathrm{La}]_{\text {inicial }}$ e $[\mathrm{La}]_{\text {final }}$ são as concentrações de lactato inicial e final estimadas pela curva polinomial de terceira ordem ([La] $\left.=a+b \cdot v+c \cdot v^{2}+d \cdot v^{3}\right)$ para as respectivas velocidades inicial (vi) e final (vf). 
Os dados são apresentados como média \pm desvio padrão (DP). Os dois métodos foram comparados através do teste $t$ de Student bicaudal pareado. A correlação entre os métodos foi feita utilizando-se o coeficiente de correlação de Pearson. Para a análise de concordância entre os métodos foi utilizada a análise de Bland-Altman (BLAND; ALTMAN, 1986). Adotou-se um nível de significância de $P<0,05$.

\section{RESULTADOS}

Os valores observados para as variáveis fisiológicas ao final do teste incremental (média \pm DP) foram os seguintes $(n=17)$ : consumo máximo de oxigênio $=56,3 \pm 5,7 \mathrm{~mL} / \mathrm{kg} / \mathrm{min}, \mathrm{RER}=1,13 \pm 0,1 \mathrm{I},[\mathrm{La}]$ pico $=7,4 \pm 2,4 \mathrm{mM}, \mathrm{PSE}$ $=18,9 \pm 1,4$ e FC máxima $=188,6 \pm 10,3$ bpm. Em todos os sujeitos foram observados pelo menos três critérios de esforço máximo.

A Tabela I apresenta os valores médios \pm desvio padrão (DP) das variáveis fisiológicas obtidas durante do teste incremental. A vLL foi estatisticamente diferente $(P<0,05)$ entre os métodos $\left(v L L_{\text {Exp }}\right.$ e $\left.v L L_{\text {Pol }}\right)$ aplicando-se o teste $t$ de Student bicaudal para amostras dependentes.

Tabela I - Valores médios \pm desvio padrão (DP) da velocidade pico (Vpico) do teste incremental e velocidade de corrida no limiar de lactato ( $v L L$ ) determinada pelo método Dmax utilizando-se o

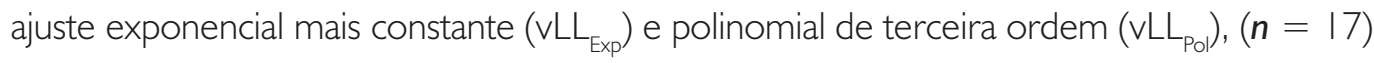

\begin{tabular}{ll}
\hline Variáveis & Média $\pm \mathrm{DP}$ \\
\hline $\operatorname{Vpico}(\mathrm{km} / \mathrm{h})$ & $13,4 \pm 1,0$ \\
$\operatorname{VL} L_{\text {Exp }}(\mathrm{km} / \mathrm{h})$ & $10,9 \pm 0,8 *$ \\
$V L L_{\text {Pol }}(\mathrm{km} / \mathrm{h})$ & $10,4 \pm 1,1$ \\
\hline
\end{tabular}

*p $<0,05$ em relação à $v L L_{P o l}$

A Figura 2 apresenta a reta de regressão (linha tracejada) entre a $v L L$ determinada pelos ajustes exponencial mais constante $\left(v L L_{\text {Exp }}\right)$ e polinomial de terceira $\operatorname{ordem}\left(v L L_{P_{0}}\right)$. O coeficiente de correlação de Pearson foi de $r=0,84(P<0,00 \mathrm{I})$ indicando uma forte correlação entre os dois métodos. As correlações entre a Vpico e as velocidades de corrida foram de $r=0,94 ; P<0,00$ I $\left(v_{L L} L_{\text {Exp }}\right)$ e $r=0,69$; $P$ $<0,00$ I $\left(v L L_{\text {Pol }}\right)$. Entre as 17 participantes, a $v L L_{\text {Pol }}$ mostrou-se apenas duas vezes ligeiramente maior que a $v L_{\text {Exp }}$ (diferença a maior de 0,06 e 0,1 I km/h). 


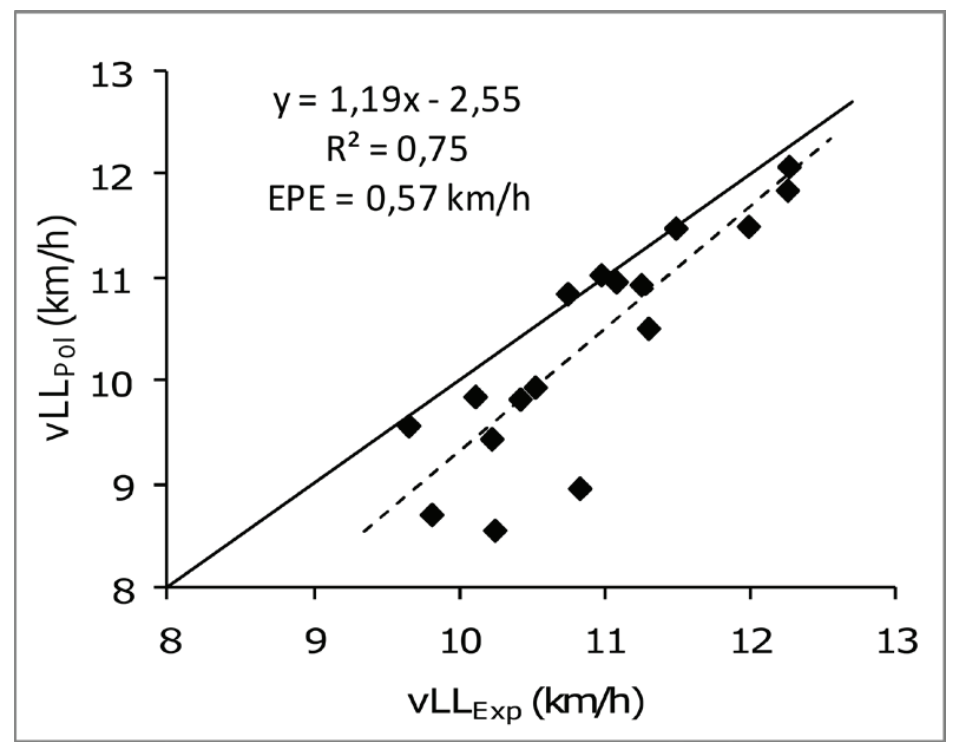

Figura 2: Reta de regressão (linha tracejada) entre a $\vee L_{\text {Exp }}$ e a $\vee L_{\text {Pol }}(r=0,84 ; P<0,00 I$ ), com a reta identidade representada pela linha contínua $(\boldsymbol{n}=17)$.

A Figura 3 apresenta a análise de concordância entre as velocidades de corrida $\vee L L_{\text {Exp }}$ e $v L L_{\text {Pol }}$ através do gráfico de Bland-Altman. A diferença entre as médias (viés) foi de 0,53 $\pm 0,57 \mathrm{~km} / \mathrm{h}$ (linha contínua), com limites de concordância (95\%) de $-0,59$ e $+1,65 \mathrm{~km} / \mathrm{h}$ (linhas tracejadas inferior e superior).

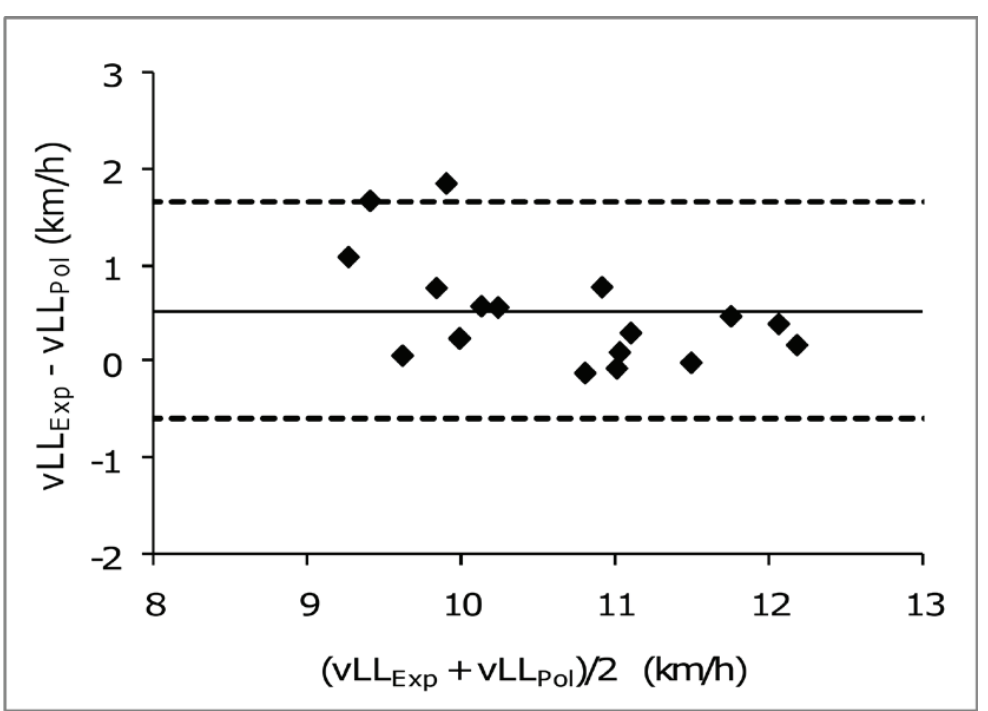

Figura 3: Gráfico de Bland-Altman $(\boldsymbol{n}=17)$ : análise de concordância entre $v L L_{\text {Exp }}$ e vLL $L_{\text {Pol }} \bigcirc$ viés foi de 0,53 \pm 0,57 km/h (linha contínua). Os limites de concordância (95\%) foram de -0,59 e + I,65 $\mathrm{km} / \mathrm{h}$ (linhas tracejadas inferior e superior).

A Figura 4 apresenta o ajuste exponencial mais constante (linha tracejada) e polinomial de terceira ordem (linha contínua) com a respectiva determinação da vLL de uma participante em que a $v L L_{\text {Pol }}(8,7 \mathrm{~km} / \mathrm{h})$ foi bem inferior a $v L L_{\text {Exp }}(9,8 \mathrm{~km} / \mathrm{h})$. 


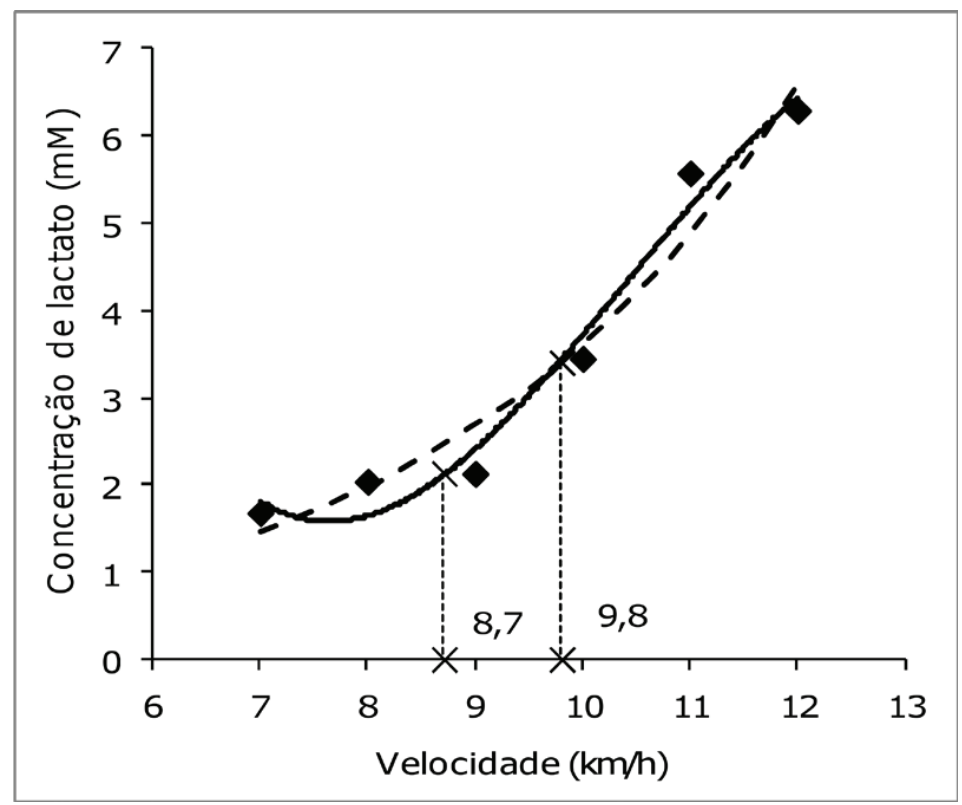

Figura 4: Ajuste exponencial mais constante (linha tracejada) e polinomial de terceira ordem (linha contínua) de uma participante. As velocidades de corrida foram de $8,7 \mathrm{~km} / \mathrm{h}\left(\mathrm{vLL} \mathrm{Pol}_{\mathrm{P}}\right)$ e $9,8 \mathrm{~km} / \mathrm{h}\left(\mathrm{vLL} \mathrm{Exp}_{\mathrm{p}}\right)$.

\section{DISCUSSÃO}

O principal achado deste estudo foi que a escolha da curva de regressão do lactato sanguíneo entre as equações exponencial mais constante e polinomial de terceira ordem influi no valor da $v L L$, sendo a $v L L_{\text {pol }}$ consistentemente inferior à $\vee L L_{\text {Exp }}$. Além disso, o limite de concordância apresentado entre a $\vee L L_{P o l}$ e a $v L L_{\text {Exp }}$ não se mostrou muito estreito, razão pela qual técnicos, atletas e pesquisadores devem evitar a utilização indistinta destes dois índices na prescrição de treinamento.

O Dmax é um método gráfico que depende tanto do formato da curva lactato-intensidade bem como das intensidades inicial e final do teste incremental. Janeba et al. (2010) verificaram que um aumento na intensidade inicial do teste incremental conduz a um aumento na intensidade do LL. Os autores verificaram também a necessidade de se realizar um teste que seja efetivamente de máximo esforço, caso contrário o LL será subestimado. $\bigcirc$ problema da intensidade inicial pode ser controlado através da aplicação e repetição de um mesmo protocolo, portanto com a mesma intensidade inicial, pré, durante e pós treinamento. $O$ problema da intensidade final, por sua vez, pode ser controlado garantindo-se o efetivo máximo esforço durante o teste incremental, tal como foi garantido neste estudo com a observação de pelo menos três dos critérios tradicionais de máximo esforço (HOWLEY; BASSETT; WELCH, 1995).

Conforme apresentado na tabela I, as velocidades de corrida foram de 10,9 $\pm 0,8\left(\mathrm{vLL}_{\text {Exp }}\right)$ e $10,4 \pm 1,1 \mathrm{~km} / \mathrm{h}\left(\mathrm{vLL}_{\mathrm{Pol}_{\mathrm{O}}}\right)$, com diferença significativa entre elas $(P<$ 
0,05). Verifica-se através da figura 2 que apesar da forte correlação entre ambos os métodos $(r=0,84 ; P<0,00 \mathrm{I})$, poucos pontos situaram-se juntos da reta identidade (linha contínua) $\left(\mathrm{VLL}_{\mathrm{Exp}}=\mathrm{vLL}_{\mathrm{Pol}} ; r=1,00\right)$. Esta dispersão de dados sugere que há discordância entre os métodos. Verifica-se também que os dados situam-se juntos ou abaixo da reta identidade, mostrando a tendência da $v L L_{\text {Exp }}$ ser maior que a $v L L_{\text {Pol }}$.

Através do gráfico de Bland-Altman (BLAND; ALTMAN, 1986) apresentado na Figura 3, confirma-se a tendência já sugerida pela Figura 2 da não concordância plena entre os métodos, com a $v L_{\text {Exp }}$, na maioria das vezes, se mostrando maior que a $v L L_{\left.P_{0}\right|^{\prime}}$ A diferença entre os métodos foi de 0,53 $\pm 0,57 \mathrm{~km} / \mathrm{h}$, com limites de concordância para 95\% dos casos (média \pm 1,96 DP) entre -0,59 e + l,65 $\mathrm{km} / \mathrm{h}$. Apesar do limite inferior de concordância ser de $-0,59 \mathrm{~km} / \mathrm{h}$, verificamos que dentre as 17 participantes, apenas duas possuíram a $v L L_{\text {Pol }}$ maior que a $v L L_{\text {Exp }}$ e ainda assim a diferença nesses dois casos foi inferior a 0, $12 \mathrm{~km} / \mathrm{h}$.

Entre os preditores do desempenho aeróbio, Noakes, Myburgh e Schall (1990) verificaram que a Vpico seguida do LL foram os índices que melhor conseguiram predizer o desempenho de corridas de 10, 21, I, 42,2 e $90 \mathrm{~km}$, obtendo correlações da ordem de 0,90 com o desempenho. Outros estudos como os de Scott e Houmard (1994) e Stratton et al. (2009) também encontraram uma alta correlação entre o desempenho em corridas de $5 \mathrm{~km}$ e a $\vee$ pico. Ainda que a $\mathrm{VLL}_{\mathrm{Pol}}$ tenha se correlacionado fortemente com a $\mathrm{VLL}_{\text {Exp }}(r=0,84 ; P<0,00 \mathrm{I})$, ela apresentou uma correlação apenas moderada com a Vpico $(r=0,69 ; P<0,00 \mathrm{I})$, ao contrário da $\vee L L_{\text {Exp }}$ que se correlacionou fortemente $(r=0,94 ; P<0,001)$, sugerindo que o ajuste exponencial mais constante se mostra mais apropriado para a predição do desempenho através do método Dmax.

Verifica-se através da Figura 4 que a configuração dos pontos pode distorcer bastante a curva polinomial de terceira ordem. Nesta figura, a concentração de lactato a 9 km/h não apresentou um aumento abrupto em relação à velocidade de $8 \mathrm{~km} / \mathrm{h}$ e, portanto, teoricamente o LL não poderia ocorrer entre essas duas velocidades tal como determinado pela $v L L_{\text {Pol }}(8,7 \mathrm{~km} / \mathrm{h})$, subestimando aparentemente - LL. Neste caso, a vLL determinada pelo ajuste exponencial $\left(\mathrm{VLL}_{\text {Exp }}=9,8 \mathrm{~km} / \mathrm{h}\right.$ ) mostrou-se mais adequada para refletir o aumento abrupto na concentração de lactato. Conforme a Figura I, apesar da distorção da curva apresentada no início da curva polinomial de terceira ordem (Figura IB), esta curva se comportou de forma semelhante à curva exponencial mais constante (Figura |A) entre as velocidades de 10 e $13 \mathrm{~km} / \mathrm{h}$, resultando em uma velocidade de corrida igual em ambos os métodos ( $\mathrm{vLL}=1 \mathrm{I}, \mathrm{I} \mathrm{km} / \mathrm{h}$ ). Desta forma, verifica-se que a disposição dos pontos nem sempre influenciará na vLL determinada pelos dois métodos. 


\section{CONCLUSÃO}

Diante do exposto, verificou-se que a escolha entre as equações exponencial mais constante ou polinomial de terceira ordem influirá na $v L L$ determinada pelo método Dmax. Além disso, a $v L L_{\text {Pol }}$ se correlacionou menos com a Vpico e mostrou tendência a subestimar a $v L_{L_{\text {xxp }}}$. Como o limite de concordância apresentado entre $a \vee L L_{p o l}$ e a $v L L_{\text {Exp }}$ não se mostrou muito estreito, técnicos, atletas e pesquisadores devem evitar a utilização indistinta destes dois índices na prescrição de treinamento. Apesar do ajuste exponencial mais constante ter se mostrado mais adequado para a determinação do LL pelo método Dmax, ainda há necessidade de comparar ambos os LL com a máxima fase estável de lactato, visto ser esta o padrão ouro para a determinação da transição do metabolismo aeróbio-anaeróbio durante o exercício.

Comparison between exponential and polynomial curve fitting on the determination of the lactate threshold by the Dmax method

ABSTRACT: The running speed at lactate threshold (VLT) determined by the Dmax method was compared using the exponential plus constant $\left(V L T_{\text {exp }}\right)$ and third level polynomial $\left(V L T_{\text {pol }}\right)$ curve fitting. Seventeen female recreational runners underwent a treadmill incremental test until voluntary exhaustion. Blood samples were collected between the stages for lactate analysis. The $v L T_{\text {exp }}(10.9 \pm 0.8 \mathrm{~km} / \mathrm{h})$ was statistically different $(P<0.05)$ from $v L T_{\text {pol }}(10.4 \pm 1.1$ $\mathrm{km} / \mathrm{h}$ ) using paired Student's $t$ test. The correlation between $v L T_{\text {exp }}$ and $v L T_{\text {pol }}$ was $r=0.84$. The correlations between peak speed and $v L T$ were $r=0.94\left(v L T_{\text {exp }}\right)$ and $r=0.69\left(v L T_{\text {po }}\right)$. It was concluded that the choice of the blood lactate regression curve influences the $\mathrm{VLT}$ value, in which the $\mathrm{VLT}$ pol was consistently lower than the $\mathrm{VLT} \mathrm{exp}_{\text {. }}$.

KEYWORDS: Equation; maximal deviation method; running speed; regression.

Comparación entre el ajuste de la curva exponencial y polinómica en la determinación del umbral de lactato por el método Dmax

RESUMEN: Se comparó la velocidad de carrera en el umbral de lactato ( $v L L$ ) determinado por el método Dmax utilizando la curva exponencial más constante $\left(V L L_{\text {exp }}\right)$ y polinómica de tercer grado $\left(V_{L} L_{p o}\right)$. Diecisiete corredoras recreativas ejecutaron una prueba incremental discontinua en la cinta rodante hasta la extenuación voluntaria con la extracción de sangre entre las etapas para el análisis de lactato. $\operatorname{La}_{\mathrm{VLL}}(10,9 \pm 0,8 \mathrm{~km} / \mathrm{h})$ fue estadísticamente diferente $(P<0,05)$ de la $v L L_{P o l}(10,4 \pm 1,1 \mathrm{~km} / \mathrm{h})$ mediante la prueba t de Student pareado. La correlación entre $v L_{E x p} y v L L_{\text {pol }}$ fue de $r=0,84$. Las correlaciones entre el pico de velocidad y las $v L L$ fueron de $r=0,94\left(v L L_{\text {exp }}\right)$ y $r=0,69\left(v L L_{\text {poo }}\right)$. Fue concluido que la elección de la curva de regresión de lactato sanguíneo influye en el valor de la $v L L$, siendo la $\checkmark L L_{\text {pol }}$ sistemáticamente inferior a la $v L L_{\text {exp }}$.

PALABRAS CLAVE: Ecuación; método de la desviación máxima; velocidad de carrera; regresión. 


\section{REFERÊNCIAS}

BILLAT, V. L. Use of blood lactate measurements for prediction of exercise performance and for control of training. Sports Medicine, Auckland, v. 22, n. 3, p. 157-175, set. 1996.

BISHOP, D.; JENKINS, D. G.; MACKINNON, L. T. The relationship between plasma lactate parameters, Wpeak and I-h cycling performance in women. Medicine and Science in Sports and Exercise, Washington, v. 30, n. 8, p. 1270-1275, ago. 1998.

BLAND, J. M.; ALTMAN, D. G. Statistical methods for assessing agreement between two methods of clinical measurement. Lancet, Londres, v. I, n. 8476, p. 307-3 I 0, fev. 1986.

BORG, G. Psychophysical bases of perceived exertion. Medicine and Science in Sports and Exercise, Washington, v. 14, n. 5, p. 377-381, set./out. 1982.

CHENG, B. et al. A new approach for the determination of ventilatory and lactate thresholds. International Journal of Sports Medicine, Stuttgart, v. 13, n. 7, p. 518-522, out. 1992.

CZUBA, M. et al. Lactate threshold (d-max method) and maximal lactate steady state in cyclists. Journal of Human Kinetics, Illinois, v. 2 I, n. I, p. 49-56, jan./jun. 2009.

DAVIS, J. A. et al. Comparison of three methods for detection of the lactate threshold. Clinical Physiology and Functional Imaging, Malmö, v. 27, n. 6, p. 38I-384, nov. 2007.

FAUDE, O.; KINDERMANN, W.; MEYER, T. Lactate threshold concepts: how valid are they? Sports Medicine, Auckland, v. 39, n. 6, p. 469-490, jun. 2009.

HOWLEY, E. T.; BASSETT JR, D. R.; WELCH, H. G. Criteria for maximal oxygen uptake: a review and commentary. Medicine and Science in Sports and Exercise, Washington, v. 27, n. 9, p. |292-|30|, set. 1995.

HUGHSON, R. L.; WEISIGER, K. H.; SWANSON, G. D. Blood lactate concentration increases as a continuous function in progressive exercise. Journal of Applied Physiology, Washington, v. 62, n. 4, p. 1975-1981, may 1987.

JANEBA, M. R. et al. The dmax method does not produce a valid estimate of the lactate threshold. Journal of Exercise Physiology Online, Duluth, v. 13, n. 4, p. 50-57, ago. 2010.

NICHOLSON, R. M.; SLEIVERT, G. G. Indices of lactate threshold and their relationship with 10-km running velocity. Medicine and Science in Sports and Exercise, Washington, v. 33, n. 2, p. 339-342, fev. 2001 .

NOAKES, T. D.; MYBURGH, K. H.; SCHALL, R. Peak treadmill running velocity during the VO2 max test predicts running performance. Journal of Sports Sciences, Londres, v. 8, n. I, p. 35-45, jan./abr. 1990.

PAPADOPOULOS, C.; DOYLE, J. A.; LABUDDE, B. D. Relationship between running velocity of 2 distances and various lactate parameters. International Journal of Sports Physiology and Performance, Champaign, v. I, n. 3, p. 270-283, set. 2006. 
SCOTT, B. K.; HOUMARD, J. A. Peak running velocity is highly related to distance running performance. International Journal of Sports Medicine, Stuttgart, v. I 5, n. 8, p. 504-507, nov. 1994.

STRATTON E. et al. Treadmill Velocity Best Predicts 5000-m Run Performance. International Journal of Sports Medicine, Stuttgart, v. 30, n. I, p. 40-45, jan. 2009.

THOMAS, $\mathrm{V}$. et al. A comparison of lactate indices during ramp exercise using modeling techniques and conventional methods. Journal of Sports Sciences, Londres, v. 26, n. I3, p. |387-|395, nov. 2008.

TOKMAKIDIS, S. P.; LÉGER, L. A.; PILIANIDIS, T. C. Failure to obtain a unique threshold on the blood lactate concentration curve during exercise. European Journal of Applied Physiology and Occupational Physiology, Berlin, v. 77, n. 4, p. 333-342, mar. 1998.

ZHOU, S.; WESTON, S. B. Reliability of using the D-max method to define physiological responses to incremental exercise testing. Physiological Measurement, Bristol, v. I 8, n. 2, p. | 45- I 54, may 1997.

Recebido: 14 out. 2010

Aprovado: 13 jun. 201 I

Endereço para correspondência:

Fabiana Andrade Machado

Depto. Educação Física, Bloco M06 - UEM Av. Colombo, 5.790 - Jd. Universitário

Maringá-PR

CEP: $87020-900$ 